\title{
Anywhere is Everywhere - Tales of a Virtual Traveller
}

\author{
Chris A. Wright \\ $\mathrm{Nb}$ Sheligoe \\ Willow Mooring, London Road, Kegworth DE74 2EY, UK \\ drcawright@yahoo.com
}

\begin{abstract}
This paper explores the notions of the virtual traveller through the physical manifestation of an exhibition Anywhere is Everywhere is a Circular Tale presented in the tAd Gallery in Denton, Texas. It is the narrative of a journey linking the eleven places called Denton in England and presents the 1,026 mile, 21 hours and 57 minute circular journey negotiated via internet-based maps. These provide the 301 steps of detailed instructions. Postcards, photographs and other ephemera from the journey are presented to form a comprehensive retelling of the tale. However, the places have only been visited virtually, the sights are seen through others' eyes, the descriptions are secondhand and the impressions gained only through what is seen on my computer screen at home. It is a tourist guide that, by way of its virtuality, creates a false reality, destroying, perhaps, a desire to visit but presenting a new sense of community.
\end{abstract}

This work can be read on two levels: firstly as a straightforward road trip linking places of the same name. The second reading of the work is one that relates to the opposition between real and virtual, its position in-between here and there and concerns of space and place, as well as exploring facets of contemporary tourism. Artistically, it relates to the ready-made and questions of authorship and value.

The circular journey to the places called Denton is a binary opposite to the notion of the flâneur. Here, the directions minutely laid out leaving no space for wandering and following interesting paths. Yet, by the motion of the viewer within the gallery space, it can again become an act of wandering. This has some similarities to the act of Internet browsing. Franklin writes 'We surf the net routinely, whizzing about the world at fantastic speeds and this does indeed cancel distance, but the point I want to make here is that we surf like tourists and the Web is set up in a touristic way' (Franklin 2003:8). Contemporary artists such as Richard Wentworth and Francis Alÿs have used the concept of the flâneur to encounter first-hand everyday activities whilst here it can be said that the wandering takes place within the exhibition space but the basis of the exhibition is a kind of Internet flâneurism. The act of viewing, and wandering, also relates to the act of walking. There seems to be a relationship with the act of walking as to how places are created. De Certeau says 'to walk is to lack a place. It is the indefinite process of being absent.' (De Certeau 1988:103). It could be seen either as the constant creation of place or that each step removes us from our 'place', from what we know, however temporarily. Questions arise as to how this aligns with the knowledge of places through the web? How does this journey, the virtual narrative of actual places align to the concept of travel as a leisure activity? Is it pleasurable, will it create along-lasting impression, a memory to be unravelled at various points of life for the benefit of others, an indulgence?

The aim in this paper is to explore these ideas through presentation of facets of the exhibition.

Computer and electronic arts. Virtual communities. Virtual cultural heritage.

\section{INTRODUCTION}

This paper explores, from a fine art perspective, the notions of virtual travel, drawing on the ideas shown in my solo exhibition Anywhere is Everywhere is a Circular Tale at tAd Gallery in Denton, Texas, USA, in 2014. It became the physical manifestation of a journey that attempted to link the eleven places called Denton, in England with Denton, Texas. It was the narrative of a road journey that was 1,026 miles long and would take 21 hours and 57 minutes to complete in a circular loop. It was planned and negotiated via internetbased maps. Exhibited at the gallery were the 
textual presentation of the 301 steps of detailed instructions, a series of souvenirs in the form of cards showing buildings that could be cut out and assembled, and eleven identical postcards created of Denton at Night. Each of these cards was separately posted to the gallery from England. The final part of the exhibition was a fast-moving, silent presentation that gave the impression of being taken from a moving vehicle. Selected images were taken from the web and carefully chosen to avoid identifiable people or vehicles and generally showed the most anodyne of places such as road junctions and bits of motorway. Each image, however, featured the memorable words Google Copyright. These objects, taken together, formed a comprehensive retelling of the tour of the Dentons.

This work can be read on two levels. Firstly, as a straightforward road trip linking the eleven places whose only apparent relationship, for ten of them at least, is the same geographical feature. The origin of the Denton located in Northamptonshire is a 'settlement associated with Dodda' whilst the others come from Old English meaning of a settlement in a valley. The name of Denton was also a fictional place for the TV series 'A Touch of Frost'.

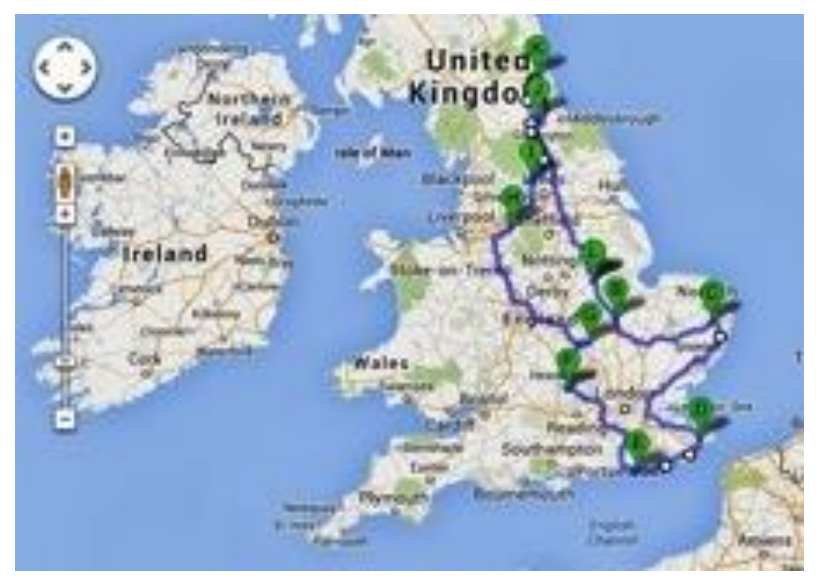

Figure 1: Map of journey

The second reading explored the work from a variety of different perspectives. Artistically, it relates to the ready-made, the representation of objects and questions of authorship and value as well as modes of viewing. From a web perspective, there were issues regarding copyright and usage as well as authenticity. Other questions related to the concept of place and placelessness, the opposition between real and virtual, its position inbetween here and there as well as exploring facets of contemporary tourism. It questions value and cultural heritage and potential advantages and disadvantages for both traveller and location.

The exhibition, which was used as a resource for this paper, was conceived from extensive research into the nature of place as well as diverse field trips to places such as Las Vegas and New Zealand where the inauthentic tourist experience of Maori heritage contributed to the concept of this exhibition. In Las Vegas, it was realised that, to the tourist, the lake inspired by Lake Como, Bellagio, Italy and re-constructed at the Bellagio Hotel, Las Vegas was as much a destination as the real thing.

The concerns that arise can be broken down into sections. Firstly, the nature of material presented, secondly, ways of travel and viewing and lastly, trust and mistrust concerning issues of authenticity and authorship.

\section{NATURE OF MATERIAL}

The basic aim was to explore how material taken from the Internet could be used to create an authentic travel experience. Questions asked included how providing pages of directions could create meaning, how the addition of 'genuine' mementos could add to the experience and whether the concept of the exhibition was a valid one.

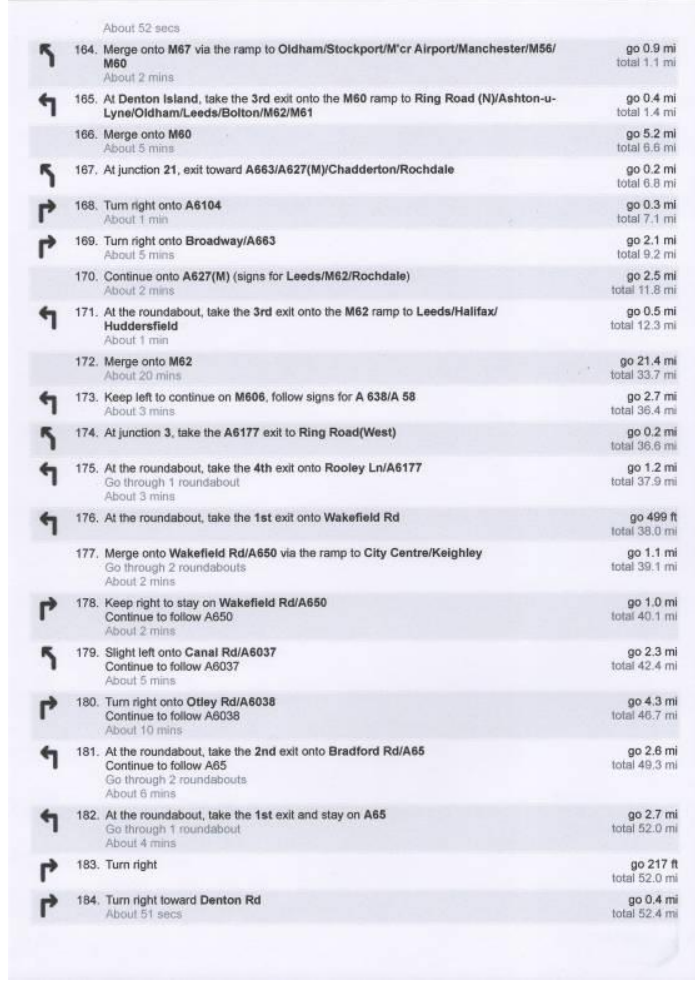

Figure 2: List of steps, sheet 10

Detailed directions were presented which showed each turn of the road in textual detail and occasionally included a map. From this information, there was little sense of distance or place, just endless steps highlighting the fact that a long journey was being undertaken. However, it conveyed no sense of the physicality of movement creating an armchair travel document. 
From each place called Denton, fictional souvenirs were created in the form of assembling models. Each showed the frontage, reflecting the 2D origin of the images, of a specific, named building from each place called Denton. The sheets include cutting out details and acknowledgment and credit of the original photographer. The buildings chosen were public buildings or locations such as guesthouses and were not representative of the places as a whole. The sheets were intended be displayed as either objects in their own right or to be cut out and folded as per instructions on the sheets.

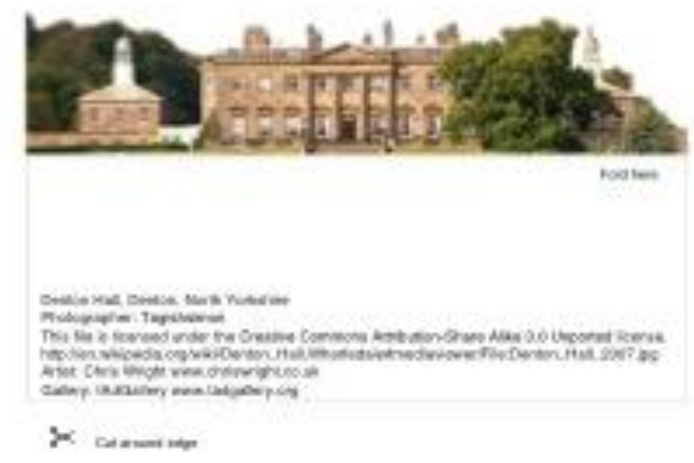

Souvenir Sheets

A New Denton

No. 9

Figure 3: Souvenir sheet No. 9

From the assembled models, a village could be created of a new Denton. Slipped amongst the models of the eleven English Dentons, was an additional sheet pertaining to Denton, Texas thus enabling a contemporary global community to be created. By changing the position of the models around, different configurations can highlight both the similarities and differences of diverse communities. Emotions and tensions that relate to class, power and authority are rendered powerless by the scale of the models. The models do not reflect the actual building size but the size of the original image creating an erratic sense of scale. For example, the larger the building, the smaller it has become due to my standardizing of image size. A model could thus could be placed to reflect the way the stately home is further away, or higher up the hill from the hoi polloi and general everyday happenings. To anarchically invert the class system, the 'big' houses can be juxtapositioned against the smallest making a community of
Denton that disregards, for example, wealth and class. It also collapses the distance that exists between them.

By creating souvenirs, I am attempting to formalize the journey and make it real. Within the model village of New Denton, a community is imagined and, maybe, idealized. The conflation of the models reflects the nature of the viewing process through web-based media where time and distance are immaterial.

In addition, postcards, printed using a web-based company, were produced that purported to be authentic mementoes from England. Postcards were chosen because, although it could be considered out-dated to send a card, tourist destinations and other places still display an abundance of postcards. Sent from England to Texas, they created a unifying link. Each of these cards showed the same image, Denton at Night making it impossible to distinguish which Denton was being referred to. The cards were handwritten, all different, with some signed creating a personal link with Texas, the only human gesture within the exhibition.

The final part of the exhibition was a presentation that showed scenes from the journey, flashing by in an endless loop. Each flash was an image that was, by necessity, low quality and often disjointed exposing their provenance. The presentation was compiled from others' web photographs or street map views from Google, the symbol of which was present in each image acknowledging its presence. The speed of the presentation means each image was almost lost in the constant motion. The roads and junctions with their endless roundabouts act as disjointed sites but are recognizable as links and nodes.

The places become lost in that moment of viewing time. They are not experienced as much as a fragile moment created. The collapse of time and space within the virtual viewing experience was mitigated by the very public showing of the journey and by the presentation of mementoes. It could also be said that, here, time was marked only by the presence of the viewer. Temporality is necessarily implicit within journeys of any kind and the act of viewing created its own journey and thus temporality. It could be said that Anywhere is Everywhere is a microcosm where time and space are conflated to become one. The succession of moments thus had a kind of vertical layering due to the making real of the virtual moment relating to the provenance of the exhibits, the virtual world and the act of viewing. 


\subsection{Ways of travel, ways of viewing}

I am sitting at home in my comfortable chair, warm and cosy, coffee to hand. There is no sound except the familiar ones of my own home, the hum of the fridge and the buzz of the fluorescent light; or I am in a gallery, surrounded by imagery, there is a low buzz of voices and a shuffling of feet. Yet, I am travelling up a motorway, cars dashing alongside. I am mesmerized by the way the scenery changes; by the way I zip along from town to country. Mansions and thatched cottages, woods and fields and changing weather patterns entrance me. But there is no relevant sound, no smells of farms, nothing to ground me in the location and into the business of sightseeing.

The notion of creating a journey through a collection of images is not a new one. Any travel agent will give you an armful of brochures highlighting the delights of particular locations. Not, however, the wonders of the local town but the hotels and their pools as if these were destinations in their own right with the actual country an added extra, an irrelevance. This is very much the experience of the virtual traveller. The places visited are already mediated through the web, through Trip Advisor or through personal blogs. The images are incongruent, a plate of food or an amusing hat aligned with a feat of engineering or an amazing price tag on a counterfeit handbag, images which are not representative of their location but of the experience of the travellers themselves. The Internet weaves a disparate web that can only be experienced disjointedly.

The act of embarking on a virtual journey is one that transgresses the very notion of travel. Travel implies the physical advance from one place to another. By the virtuality of this journey, the progress requires imagination for forward movement. The circularity of Anywhere is Everywhere could be said to provide a distasteful answer to the child's question 'are we there yet'? You are never there. In addition, you were never there, you were here and will remain so. This accurately defines virtual travel. Franklin writes:

\footnotetext{
'We surf the net routinely, whizzing about the world at fantastic speeds and this does indeed cancel distance, but the point I want to make here is that we surf like tourists and the Web is set up in a touristic way' (Franklin 2003:8).
}

This way of travelling becomes a different encounter, a transaction between mediated images. In a gallery, everyone is a tourist.

To compare the act of net surfing to walking around any gallery where the journey is spatially finite, the gallery has to become a space of wonder. It has to entice so that exhibits attract irresistibly, creating a meandering route, a psychogeographical journey rather than a logical direction, or the direction the curator wishes you to undertake. This is in opposition to the journey undertaken of the Dentons where the route is laid out in minute detail. Each turn specified, an order with no place for the flâneur. Therefore, to wander around tAd Gallery amongst the instructions and finite details of Anywhere is Everywhere, as opposed to following logically, creates a new situation, which I believe can be seen as place making. De Certeau says,

\section{'To walk is to lack a place. It is the indefinite process of being absent' (De Certeau 1988:103).}

This could be seen as each step temporarily removing us from our 'place', from what we know, In addition, I see walking, especially wandering, as the constant creation of place. To create place, an event has to occur and this could be allied with the viewing process, with the act of wonder and wandering. In essence, in this exhibition, there is a continual dichotomy between definitions of place as per exhibit. That is, what we are viewing and our manner of viewing it. Viewing on the Internet happens in the viewers' real time, we act as individuals in our own private space; but just press pause and I'll make coffee; even though this space is shared virtually. When viewing in a gallery situation, you are going to a place where there are others like you, entering a known place. Experience is shared by individuals but individuals that act complicitly due to rules and regulations imposed by the institution and adhered to due to peer pressure.

The ways of experiencing the journey shown in this exhibition reveals a different way of looking that takes account of technological innovation. Barbara London, video curator at the Museum of Modern Art, New York, writes:

\footnotetext{
'The assurance that time flows in an unending regular manner no longer applies. Time must now conform to a menu of future events' (London 1995:423).
}

The 'future' of time therefore has to be visualised according to the technological events that may occur but these may not yet be known. Time is a way of negotiating space that is implicit both within that negotiation itself and also within the accessing of that negotiation. It is also true that space is a way of negotiating time.

\subsection{Trust/mistrust}

The facts presented in Anywhere is Everywhere have not been checked. Facts have been accepted as they stand reflecting how the Internet is commonly accepted with oft-misguided trust. So the whole presentation is one of questionability and 
is quite possibly purely fictional. The aim here was to use that trust/mistrust as a starting point for how complete fictions could be manufactured whereby, whether true or false, their outward appearance looks genuine. Adrian Franklin states,

\begin{abstract}
'We accept that everything has a claim to authenticity; everything has a unique cultural content, even fakes...It is a world of fakes, reproductions, fusions, hybrids and so forth and computer-driven simulation electronics enable (almost) everyone to be quite creative within this graphical, visual infinity' (Franklin 2003:266).
\end{abstract}

The authenticity of the souvenirs, in fact, of any souvenirs in the global world of counterfeit, has to be questioned, but in the context of this exhibition are presented as authentic. This debate about authenticity has existed for a long time and a conclusion of sorts has been generally reached that everything is authentic within its own context. The 'fake' souvenirs presented here are no more false than say, souvenirs of England made abroad or in places such as China. The authenticity of the whole exhibition is based on the artist manipulating information and artefacts to present a coherent account of a fictional journey. Where does art lie, is it fact or fiction? Is it in the images shown as the travelogue where no attempt is made to hide the origins?

A question posed by the 2005 exhibition Universal Experience: Art, Life and the Tourist's Eye at the Museum of Contemporary Art in Chicago asks:

'What kind of truth do we want to believe in?' (Bonami and Dahlgren 2005:18).

The truth here could be seen as a new kind of authenticity whereby representation is no longer an accurate simulacra but a haziness of vision that intersects past and present, time and space and mediates between real and false, constantly weaving in and out.

To accept that there may be validity to the blurring of boundaries with regard to authenticity, however, opens up more concerns about authorship. Who owns what? One view is that to place something in the public domain, one relinquishes any authority one may have had over it. It may be that we have to concur that the public/private debate is blurred, that what we own is no longer clear-cut. Of the factors that contribute to this debate such as economics and editing, it is the misuse of images/material that causes harm in some way that is the most problematic.

\section{CONCLUSION}

The exhibition Anywhere is Everywhere is a Circular Tale at tAd Gallery, Texas, USA was used as a resource for exploring different aspects of visual and media-based arts and their relationship with the viewer. Questions asked included how could visual arts be physically represented through the medium of the web, how using the notion of a virtual journey could relate to the reality of a physical journey, how aspects of authenticity could be manipulated by the artist to create a viewing experience, what were the questions that related to authorship of the work when the work was sourced from numerous sites that composed of publicly placed imagery and, importantly, how a viewer related to this exhibition and what was the conclusion of that viewing experience?

Could it be that virtual travel may actually provide a strategy for future journeys? Restructuring the space of travel would have some advantages for both traveller and location. Whilst obvious disadvantages include the fact that the virtual visitor doesn't gain any understanding of a different culture or language and the location doesn't benefit from increased income, there are advantages. For the home-based traveller, it would provide affordable, safe, time saving and time limitless travel whilst for the location it would be environmentally friendly, save the need to provide specific infrastructure and be culturally protected from outside influence. So for the virtual visitor, no tummy upsets, for the holiday place, no drunken hen parties.

The experiences of the physical visitors to this exhibition were apparently ambiguous. According to the curator, Araya Vivorakij, there were, as expected, many viewpoints, one of which was an outright dismissal of the exhibition in general. Another was disappointment that Denton, Texas was not unique, that there were so many and especially as an organisation exists called Only in Denton, based in Texas. A contrasting reaction was a fascination by the existence of the other places and much time spent examining the artefacts. This calls into question the ethics of an exhibition based on inauthentic experience, as it may not have occurred to these viewers that the artefacts were not necessarily truthful.

Although I have tried to answer the numerous questions that have been posed by this exhibition and its material, subsequent points of discussion have occurred during the writing of this paper. One of the most important, though, concerns the response of web users and their belief in what they see and read which affects all parts of life, and art. 


\section{REFERENCES}

London, B. (1995) Time as medium. Leonardo 28(5), 423.

Bonami, F. \& Dahlgren, K. (2005) Universal experience: art, life and the tourist's eye: published in conjunction with the exhibitions at the Museum of Contemporary Art, Chicago, 12 February - 5 June 2005, Hayward Gallery, London, 6 October - 11 December 2005 and Museo d'Arte Moderna e Contemporanea di Trento e Roverto, 10 February 14 May 2006. Chicago: Museum of Contemporary Art, USA.

De Certeau, M. (1988) The Practice of Everyday Life. Berkeley, Calif.; London: University of California Press.

Franklin, A. (2003) Tourism: An Introduction. Sage Publication.

\section{RESOURCES}

Buchanan, I. (2010) A Dictionary of Critical Theory. Oxford: Oxford University Press.

Larsen, L. (2014) Networks Whitechapel Gallery and MIT Press.

Meethan, K. (2001) Tourism in a Global Society. Basingstoke: Palgrave.

Wright, C. A. (2012) The Presence of Absence and Other States of Space. PhD thesis.

http://www.ancestry.co.uk/nameorigin?surname $=$ denton

(retrieved 7 September 2014).

http://www.domesdaybook.co.uk

(retrieved 7 September 2014).

http://en.wikipedia.org/wiki/Denton

(retrieved 7 September 2014).
http://en.wikipedia.org/wiki/Denton, Lincolnshire (retrieved 7 September 2014).

http://parishes.lincolnshire.gov.uk/Denton/ (retrieved 7 September 2014).

http://www.denton-norfolk.co.uk (retrieved 7 September 2014).

http://en.wikipedia.org/wiki/Denton Cambridgeshire (retrieved 7 September 2014).

http://en.wikipedia.org/wiki/Denton, Kent (retrieved 7 September 2014).

http://en.wikipedia.org/wiki/Denton, East Sussex (retrieved 7 September 2014).

http://en.wikipedia.org/wiki/Denton, Oxfordshire (retrieved 7 September 2014).

http://www.denton-village.com/news-3/informationfrom-the-police/ (retrieved 7 September 2014).

http://en.wikipedia.org/wiki/Denton, Greater Manc hester (retrieved 7 September 2014).

http://www.genuki.org.uk/big/eng/LAN/Denton/ (retrieved 7 September 2014).

http://www.geograph.org.uk/gridref/SJ9250095400 (retrieved 7 September 2014).

http://en.wikipedia.org/wiki/Denton North Yorkshir e (retrieved 7 September 2014).

http://en.wikipedia.org/wiki/John Marley (geologist L (retrieved 7 September 2014).

http://en.wikipedia.org/wiki/Denton, Newcastle upo n Tyne (retrieved 7 September 2014).

http://www.tadgallery.org (retrieved 14 May 2014). 